\title{
Spacetime contraction in the Einstein's elevator
}

\author{
Antonio Feoli*10 \\ ${ }^{1}$ University of Sannio, Department of Engineering, Piazza Roma 21, 82100, Benevento, Italy.
}

Received on August 11, 2020. Revised on September 12, 2020. Accepted on September 15, 2020.

\begin{abstract}
The equivalence principle was explained by Einstein using the didactic "mental experiment" of a uniform gravitational field inside an elevator. The Earth gravitational field is not really uniform so the question about how to create a uniform gravitational field is legitimate. To this aim, instead of using metrics depending on spatial coordinates, we have studied a cosmological - like metric and we have found that, under suitable assumptions, it is possible to measure a uniformly accelerated motion for the test particles moving inside the elevator.
\end{abstract}

Keywords: General Relativity, Equivalence principle.

\section{Introduction}

Albert Einstein constructed his General Relativity starting from two postulates: the equivalence principle and the principle of general covariance. Before and after the enormous success of his theory these two principles were the subject of studies and discussions. Still today, after more than one century, the debate about their interpretation, application and generalization is very fertile. We have already written a pedagogical review [1], but a lot of articles have been published in particular about the violations of the Equivalence Principle. We have devoted two previous papers to that subject, one from the classical point of view [2], and the other one [3] for quantum regime. Different forms of this principle are used in textbooks, but we prefer the simple definition given by Pauli "In Newtonian theory, a frame of reference located in a homogeneous gravitational field is perfectly equivalent, from a mechanical point of view, to a uniformly accelerated reference frame. The request that all physical processes, in both systems, happen according to the same laws is the Einstein equivalence principle" [4]. In order to explain his idea, Einstein invented the gedankenexperiment of the elevator. An observer in an elevator cannot tell whether he and the elevator are uniformly accelerated in the free - field space (for example placing the elevator inside an accelerating rocket) or whether the elevator is at rest in a homogeneous gravitational field. Of course a common gravitational field, as the one generated by the Earth, is not really homogeneous and violations of the equivalence between the two reference frames occur, even if the smaller the elevator, the most negligible the magnitude of the violation [2].

On journals devoted to the didactic of physics, the debate about the construction of a real uniform gravita-

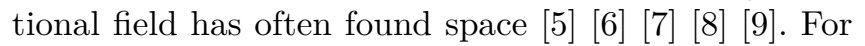
example, in a series of papers, Desloge [5] 6] (7) faced

*Correspondence email address: feoli@unisannio.it the problem showing that $[5]$ the "observations made inside a rigid enclosure at rest in a uniform gravitational field are not identical to observations made inside a rigid enclosure uniformly accelerating in field - free space" He studied the properties of a metric

$$
d s^{2}=e^{2 g x / c^{2}} c^{2} d t^{2}-d x^{2}
$$

describing a homogeneous gravitational field and compared the results with the behavior of a particle in an accelerated frame of reference (where $g$ is the constant acceleration) in the absence of a gravitational field:

$$
d s^{2}=\left(1+\frac{g x}{c^{2}}\right)^{2} c^{2} d t^{2}-d x^{2}
$$

In order to give an answer to the same problem faced by Desloge, we want to take a different approach and to construct a metric similar to the one used in cosmology. Instead of studying metrics depending on space coordinates, as it has been done until now, our idea is to explore metrics depending on the time coordinate. In general a massive body and the floor of the enclosure become closer and closer, either because the body falls towards the floor attracted by an external mass (in the elevator on Earth), or because the floor moves towards the body accelerated by an external force (in the rocket in the space far from any massive object). In principle, a third way can exist: the space between the floor and the body can disappear if it undergoes a suitable contraction, that is the contrary of the cosmological expansion. We want to analyze this theoretical possibility suggesting a metric that can simulate the effects of a uniform gravitational field. Regardless of the final result, from the didactic point of view, it may be enough to face the problem of defining the properties of a uniform gravitational field and to underline the differences with respect to the space of uniformly accelerated observers, which we do in section 2 and 3 of the paper. In section 2 we in fact summarize the dynamics of a uniformly accelerated test particle and 
the properties of the corresponding Rindler spacetime. In section 3 we describe the assumptions necessary to obtain a uniform gravitational field. Furthermore, in section 4 , we find a metric that, under suitable assumptions, simulates the required effects and in section 5 we discuss the obtained results.

\section{Rindler Flat Spacetime and its Generalization to Curved Space}

A body moving with a constant acceleration in a flat spacetime describes a hyperbolic trajectory. This fact was first noted by Minkowski [10] and then by Born [11], but today the metric of uniformly accelerated observers is known as Rindler Spacetime. Rindler himself suggested a generalization of this hyperbolic motion even to curved spacetimes, applying his results to Schwarzschild and de Sitter spacetimes 12 and then studying the relation with Kruskal Space 13. Briefly his analysis starts considering a particle's world-line $x^{\mu}(\tau)$ and defining the corresponding velocity $U^{\mu}=d x^{\mu} / d \tau$ and acceleration $A^{\mu}$ built through covariant derivative (for a definition see Appendix) with respect to the proper time of the accelerated observer $\tau$

$$
A^{\mu}=\frac{D U^{\mu}}{d \tau}=\frac{d^{2} x^{\mu}}{d \tau^{2}}+\Gamma_{\nu \sigma}^{\mu} \frac{d x^{\nu}}{d \tau} \frac{d x^{\sigma}}{d \tau}
$$

Then he studies the dynamics of a uniformly accelerated frame such that

$$
A^{\mu} A_{\mu}=-\alpha^{2}=\text { constant }
$$

that is with a non vanishing covariant acceleration.

Rindler shows that the hyperbolic motion in curved spacetime satisfies the equation

$$
\frac{D A^{\mu}}{d \tau}=\frac{\alpha^{2}}{c^{2}} U^{\mu}
$$

with the solution

$$
U^{\mu}=c\left(\cosh \frac{\alpha \tau}{c}\right) L^{\mu}+c\left(\sinh \frac{\alpha \tau}{c}\right) M^{\mu}
$$

that represents the four velocity of the uniformly accelerated test particle (the four-vectors $L^{\mu}$ and $M^{\mu}$ are such that $D L^{\mu} / d \tau=D M^{\mu} / d \tau=0, L^{\mu} L_{\mu}=1, M^{\mu} M_{\mu}=-1$ and $\left.L^{\mu} M_{\mu}=0\right)$.

In curved spacetime one must find the right expression of these two four-vectors, while in flat spacetime they assume the trivial form $L^{\mu}=(1,0,0,0)$ and $M^{\mu}=$ $(0,1,0,0)$. According to different initial conditions one can find slightly different transformations which relate the coordinates $(\mathrm{t}, \mathrm{x})$ of the inertial frame with the proper time $\tau$ of the accelerated observer. From (6) the simplest solution is:

$$
t(\tau)=\frac{c}{\alpha} \sinh \left(\frac{\alpha \tau}{c}\right)
$$

and

$$
x(\tau)=\frac{c^{2}}{\alpha} \cosh \left(\frac{\alpha \tau}{c}\right)
$$

In terms of the coordinate of Minkowski spacetime the velocity of the uniformly accelerated particle (equation (8) of ref. [12]) is

$$
V=\frac{\alpha t}{\sqrt{1+(\alpha t / c)^{2}}}
$$

We can obtain the corresponding Rindler space using the parametrization:

$$
\xi=\frac{c}{\alpha} ; \eta=\frac{\alpha \tau}{c} ; \xi \eta=\tau
$$

so that from the equations (7) and (8)

$$
\begin{aligned}
t & =\xi \sinh \eta \\
\frac{x}{c} & =\xi \cosh \eta
\end{aligned}
$$

and write the Minkowski metric in the form

$$
d \tau^{2}=\xi^{2} d \eta^{2}-d \xi^{2}-d y^{2}-d z^{2}
$$

that was called Rindler metric. We can immediately calculate the corresponding Christoffel symbols of the second kind [14 15]

$$
\Gamma_{00}^{1}=\xi ; \quad \Gamma_{10}^{0}=\Gamma_{01}^{0}=\frac{1}{\xi}
$$

Note that in Rindler coordinates the components of covariant acceleration are

$$
A^{\mu}=\left(0, \frac{1}{\xi}, 0,0\right)=(0, \alpha, 0,0)
$$

satisfying the equation (4) and the components of the Riemann tensor are vanishing so that the spacetime is flat.

\section{The Properties of a Uniform Gravitational Field}

In the spirit of the Equivalence Principle we want to construct a spacetime geometry that in the Einstein elevator induces a uniform gravitational field whose effects are equivalent to the ones measured by an observer in a uniformly accelerated frame. To this aim it is not enough to require that the final equation of motion for a massive body falling in the uniform gravitational field is the same as equation (9) of the previous section measured by the observer in the accelerated frame. Actually we must also require that the uniform gravitational field is the effect of a real curvature of spacetime, so there are nonzero components of the Riemann tensor (the contrary of Rindler spacetime) and, as a consequence, a free falling body, moving in this field, follows a geodesic, so its covariant acceleration must be zero (the contrary of Rindler spacetime).

Furthermore, we must also assume that our ideal elevator is a "rigid body" (even if we know [16] the "difficulties encountered in the search for a satisfactory definition of rigidity in relativity") and its size does not change if it is embedded in whatever gravitational field. 


\section{The Cosmological-Like Metric}

Now we choose a particular form of the metric that, following the idea explained in the introduction, can generate a contraction of the spacetime in only one direction (for example the $x$-axis) due to a sort of scale factor that plays a role opposite to the one of the cosmological expansion:

$$
d s^{2}=c^{2} d t^{2}-S(t)^{2} d x^{2}-d y^{2}-d z^{2}
$$

and we calculate (see Appendix) the corresponding Christoffel symbols of the second kind 14,17

$$
\Gamma_{11}^{0}=\frac{S \dot{S}}{c} ; \quad \Gamma_{10}^{1}=\Gamma_{01}^{1}=\frac{\dot{S}}{c S}
$$

where a dot denotes a derivative with respect to the time $t$. We argue that the geometry is a good candidate to describe a curved spacetime (first requirement), because the Riemann tensor has, for example, a non vanishing component

$$
R_{001}^{1}=\frac{\ddot{S}}{c^{2} S}
$$

In order to satisfy the second requirement we calculate the components of the four velocity that are solutions of the geodesic equations for this metric and that make all vanishing the components of the covariant acceleration. The geodesic equations are:

$$
\begin{aligned}
& A^{0}=\frac{D u^{0}}{d s}=\frac{d^{2} x^{0}}{d s^{2}}+\Gamma_{11}^{0} \frac{d x^{1}}{d s} \frac{d x^{1}}{d s}=0 \\
& A^{1}=\frac{D u^{1}}{d s}=\frac{d^{2} x^{1}}{d s^{2}}+2 \Gamma_{01}^{1} \frac{d x^{0}}{d s} \frac{d x^{1}}{d s}=0
\end{aligned}
$$

that are satisfied (as in the cosmological case) by a geodesic field comoving with the particles

$$
u^{\mu}=\frac{d x^{\mu}}{d s}=(1,0,0,0)
$$

In this case we have particles that recede from (or approach to) each other only because of the scale factor $S(t)$.

As in the cosmological metric, in our space-time the physical distance is defined as

$$
D=\sqrt{S(t)^{2} x(t)^{2}+y_{0}^{2}+z_{0}^{2}}=S(t) x(t)
$$

if we put for simplicity $y_{0}=z_{0}=0$. The corresponding "physical velocity" is

$$
\frac{d D}{d t}=\dot{S} x+S \dot{x}=H D+S \dot{x}
$$

The last term is often called peculiar velocity

$$
V_{p e c}=S(t) \dot{x}(t)
$$

and it is vanishing for the geodesic field (21). The simplest choice is $S(t)=\left(K-g t^{2} / 2\right) / \ell$ (where $K$ and $\ell$ are constants with dimension of a length) that gives the right uniform acceleration field. However it causes a problem in the corresponding $D(t)$ and its initial conditions: the constant $\mathrm{K}$ would acquire a different value for each different initial position of the falling body. The metric would depend on the initial space coordinate.

Hence we follow another approach, we assume that $u^{1}$ is non vanishing. In this case the solution of the two geodesic equations (19) and (20) is:

$$
u^{\mu}=\frac{d x^{\mu}}{d s}=\left(\frac{\sqrt{S^{2}+1}}{S}, \pm \frac{1}{S^{2}}, 0,0\right)
$$

Hence

$$
\frac{d x}{c d \tau}=\frac{d x}{d t} \frac{d t}{c d \tau}=\frac{d x}{d t} \frac{\sqrt{S^{2}+1}}{S}=\frac{1}{S^{2}}
$$

This way the peculiar velocity is

$$
V_{p e c}=S(t) \dot{x}= \pm \frac{c}{\sqrt{S^{2}+1}}
$$

Choosing

$$
S(t)=\frac{c}{g t}
$$

we obtain the typical velocity (9) of a uniformly accelerated field

$$
V_{p e c}= \pm \frac{g t}{\sqrt{1+(g t / c)^{2}}}
$$

Taking into account also the cosmic contraction, the velocity becomes

$$
V=\frac{d D}{d t}=\frac{\dot{S}}{S} D \pm \frac{c}{\sqrt{S^{2}+1}}
$$

so for each point inside the elevator the local velocity (the one measured by a static (21) observer that sees the particle to pass near $\operatorname{him} \mathrm{D}=0$ ) is the right one of a uniformly accelerated motion (29). There is also another argument, found in several textbooks about cosmology (see for example [18]), that leads to a similar result. We consider two nearby comoving observers in $\mathrm{P}$ (position at a distance $d$ from the floor)) and $\mathrm{Q}$ (position of the floor). A test particle passes observer $\mathrm{P}$ with a peculiar velocity toward Q. If $\mathrm{P}$ and $\mathrm{Q}$ are close enough that $V_{p e c}>>V_{H}$ their separation remains almost constant for the time $t_{P Q}=d /\left|V_{p e c}\right|$ of travel toward Q. But relatively to the observer Q the velocity of the particle is grater $V_{\text {pec }}+V_{H}$ and it is increased at a rate

$$
\frac{d V_{p e c}}{d t}=-\frac{d\left|V_{p e c}\right|}{d t}=\frac{H d}{t_{P Q}}=\frac{\dot{S}}{S}\left|V_{p e c}\right|
$$

Integrating we obtain $\left|V_{p e c}\right| \propto S^{-1}$ that is, using (28), $V_{\text {pec }} \simeq-g t$, the required velocity for a uniform gravitational field.

In order to avoid the divergence of the function $S(t)$ for $t=0$, it is possible to add a small constant $\epsilon$ in the equation (28) so (29) becomes

$$
V_{p e c}= \pm \frac{\epsilon+g t}{\sqrt{1+(\epsilon+g t)^{2} / c^{2}}}
$$


The class of geodesics we have found simulates well a uniform gravitational field, when $u^{1}$ of the test particle is nonvanishing. In conclusion, we obtain our goal with a metric of this kind:

$$
d s^{2}=c^{2} d t^{2}-\frac{c^{2}}{(\epsilon+g t)^{2}} d x^{2}-d y^{2}-d z^{2}
$$

but it simulates the effects of a uniform gravitational field only under suitable assumptions, not in every possible case.

Finally we examine the point of view of an observer standing on the floor of the elevator. In the Einstein gedanken experiment the elevator, in the Earth field, is not free falling but is a rigid structure fixed at rest by non gravitational forces that annul the gravitational field. Hence, there are two possible scenarios. In the first, the non gravitational forces acting on the floor can be chosen such as to make the Hubble velocity vanishing. In this case only the peculiar velocity survives and the motion of a body with respect to the floor becomes automatically uniformly accelerated. In the second case, we can imagine that the elevator must keep the distance $h$ between the floor and the ceiling constant, through external forces that are opposite to the cosmic contraction. In particular, there will be an expansion acting on the floor with a velocity $V_{w}=H h / 2$ and an equal expansion acting on the ceiling. This way the velocity measured by the observer at rest with the floor of the elevator will be

$$
\begin{aligned}
& V=\frac{\dot{S}}{S}(D-h / 2)-\frac{c}{\sqrt{S^{2}+1}}=-\frac{g}{\epsilon+g t}(D-h / 2) \\
& -\frac{\epsilon+g t}{\sqrt{1+(\epsilon+g t)^{2} / c^{2}}}
\end{aligned}
$$

The first term becomes negligible if the elevator is small $(h \rightarrow 0)$ (an approximation analogue to the one that makes the Earth field similar to a uniform gravitational field) and if the time increases. In that case the velocity reduces to the desired one (32).

\section{Conclusions}

We have shown that a uniform gravitational field in an Einstein's elevator, can be obtained starting from a cosmological - like metric that generates a spacetime contraction. We have found a solution of geodesic equations that leads to the right peculiar velocity for the particles inside the elevator if a suitable choice of the scale factor is made. Of course, the problem to really construct this field remains still open. Using the words of Desloge [7]: "Whether or not there exists a matter distribution that would actually produce this gravitational field in some region of space is a separate problem" that could be faced in a forthcoming paper.

\section{Acknowledgments}

The author wishes to thank Giorgio Papini and Maurizio Capriolo for useful discussions. This work was partially supported by research funds of the University of Sannio.

\section{Appendix}

In this appendix we collect some useful definitions and formulas of differential geometry that are well explained in the already cited textbooks [14 [15 [17] and are often used also in interesting papers published in journals devoted to Didactic of Physics such as, for example, 19. 20].

In order to define a suitable generalization of derivative in a curved spacetime, the comparison between two vectors must be performed at the same point of the spacetime. In other words, it is necessary to transport one of the two vectors to the position of the other one. A vector field is parallel transported along a curve when it does not change the angle with the tangent vector to the curve. In curvilinear coordinates the parallel displacement from a point $P$ in $x^{\mu}$ to a point $P^{\prime}$ in $x^{\mu}+d x^{\mu}$ (Fig. 1) produces a change in the vector $A^{\mu}$ by the amount $\delta A^{\mu}$ that depends linearly on the infinitesimal displacement $d x^{\mu}$ and on the components $A^{\mu}$ itself:

$$
\delta A^{\mu}=-\Gamma_{\alpha \beta}^{\mu} A^{\alpha} d x^{\beta}
$$

where $\Gamma_{\alpha \beta}^{\mu}$ are coefficients depending on the spacetime coordinates.

Consequently, in $P^{\prime}$ we can compare the two vectors $A^{\mu}+d A^{\mu}=A^{\mu}\left(x^{\mu}+d x^{\mu}\right)$ and $A^{\mu}+\delta A^{\mu}$ corresponding to the parallel transport of $A^{\mu}$ from $\mathrm{P}$ to $P^{\prime}$. We have

$$
D A^{\mu}=A^{\mu}+d A^{\mu}-\left(A^{\mu}+\delta A^{\mu}\right)=d A^{\mu}-\delta A^{\mu}
$$

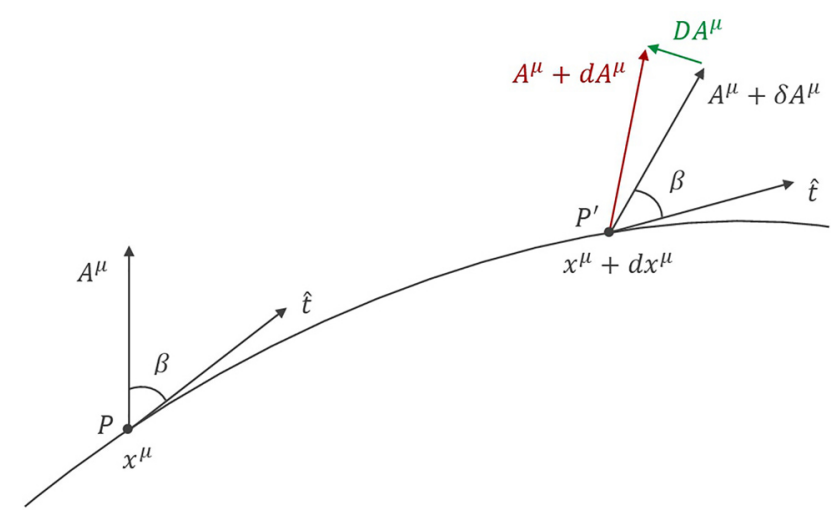

Figure 1: A vector field is parallel transported along a curve from a point $P$ to an infinitesimal close point $P^{\prime}$ leaving the angle $\beta$ with the tangent unit vector $\hat{t}$ to the curve unchanged. Only when it is in the point $P^{\prime}$, it can be compared with the vector $A^{\mu}+d A^{\mu}$. 
and the covariant derivative is defined as

$$
\begin{aligned}
& \frac{D A^{\mu}}{D x^{\nu}}=\lim _{d x^{\nu} \rightarrow 0} \frac{A^{\mu}\left(x^{\mu}+d x^{\mu}\right)-A^{\mu}\left(x^{\mu}\right)-\delta A^{\mu}}{d x^{\nu}} \\
& =\frac{\partial A^{\mu}}{\partial x^{\nu}}+\Gamma_{\alpha \nu}^{\mu} A^{\alpha}
\end{aligned}
$$

In Special Relativity the equation of motion of a free particle is

$$
\frac{d^{2} x^{\mu}}{d \tau^{2}}=0
$$

whose consequence is that the particle moves on a straight line. In General Relativity we can define a covariant acceleration deriving the four velocity $u^{\mu}$ with respect to the proper time $\tau$ using the equation (36), hence the generalization of equation (38) is

$$
\frac{D u^{\mu}}{d \tau}=\frac{d u^{\mu}}{d \tau}-\frac{\delta u^{\mu}}{d \tau}=\frac{d^{2} x^{\mu}}{d \tau^{2}}+\Gamma_{\alpha \beta}^{\mu} \frac{d x^{\alpha}}{d \tau} \frac{d x^{\beta}}{d \tau}=0
$$

The result is that a particle, embedded in a gravitational field, but in absence of other external forces, moves following a geodesic in spacetime. The geodesic equation (39) can also be obtained by a minimal action principle

$$
\delta \int d s=\delta \int \sqrt{g_{\alpha \beta} d x^{\alpha} d x^{\beta}}=0
$$

hence the corresponding trajectory of the particle is the shortest path between two given points, the same property of a straight line in flat spacetime. On the contrary, in Rindler space the covariant acceleration (39) is non vanishing and there is a hyperbolic motion due to the action of an external force.

Finally, the metric tensor used in section 4 is:

$$
g_{\mu \nu}=\left(\begin{array}{cccc}
1 & 0 & 0 & 0 \\
0 & -S^{2}(t) & 0 & 0 \\
0 & 0 & -1 & 0 \\
0 & 0 & 0 & -1
\end{array}\right)
$$

while the tensor used in standard cosmology for the flat space case is

$$
g_{\mu \nu}=\left(\begin{array}{cccc}
1 & 0 & 0 & 0 \\
0 & -S^{2}(t) & 0 & 0 \\
0 & 0 & -S^{2}(t) & 0 \\
0 & 0 & 0 & -S^{2}(t)
\end{array}\right)
$$

The Christoffel symbols of the second kind can be calculated using the formula

$$
\Gamma_{\mu \nu}^{\lambda}=\frac{1}{2} g^{\lambda \sigma}\left(\frac{\partial g_{\nu \sigma}}{\partial x^{\mu}}+\frac{\partial g_{\sigma \mu}}{\partial x^{\nu}}-\frac{\partial g_{\mu \nu}}{\partial x^{\sigma}}\right)
$$

and the Riemann curvature tensor is

$$
R_{\mu \nu k}^{\lambda}=\frac{\partial \Gamma_{\mu k}^{\lambda}}{\partial x^{\nu}}-\frac{\partial \Gamma_{\mu \nu}^{\lambda}}{\partial x^{k}}-\Gamma_{\sigma k}^{\lambda} \Gamma_{\mu \nu}^{\sigma}+\Gamma_{\sigma \nu}^{\lambda} \Gamma_{\mu k}^{\sigma}
$$

\section{References}

[1] A. Feoli, Science \& Philosophy 8, 21 (2020).

[2] E. Benedetto and A. Feoli, Eur. J. Phys. 38, 055601 (2017).

[3] A. Feoli, E. Benedetto and F. Feleppa, Eur. J. Phys. 40, 025401 (2019).

[4] W. Pauli, Theory of relativity (Pergamon Press, London, 1958).

[5] E.A. Desloge, Am. J. Phys. 57, 1121 (1989).

[6] E.A. Desloge, Am. J. Phys. 57, 598 (1989).

[7] E.A. Desloge, Am. J. Phys. 58, 856 (1990).

[8] C. Semay, Eur. J. Phys. 27, 1157 (2006).

[9] G. Muñoz, P. Jones, Am. J. Phys. 78, 377 (2010).

[10] H. Minkowski, Physik. Z. 10, 104 (1909).

[11] M. Born, Ann. d. Phys. 30, 1 (1909).

[12] W. Rindler, Phys. Rev. 119, 2082 (1960).

[13] W. Rindler, Am. J. Phys. 34, 1174 (1966).

[14] S. Weinberg, Gravitation and cosmology (John Wiley \& Sons, Inc., New York, USA, 1972).

[15] M. Gasperini, Relatività Generale e Teoria della Gravitazione (Springer, Milan, 2015).

[16] W. Rindler, Am. J. Phys. 29, 365 (1961).

[17] J.V. Narlikar, Introduction to Cosmology (Cambridge University Press, 1993).

[18] L.S. Sparke and J.S. Gallagher, Galaxies in the Universe: an introduction (Cambridge University, London, 2000), $3^{\mathrm{a}}$ ed.

[19] C.R. Caraca, Rev. Bras. Ens. Fis. 39, e4301 (2017).

[20] R.F. Santos, A.C.A. Faria Junior and S.C. Ulhoa, Rev. Bras. Ens. Fis. 42, e20190003 (2020). 